\title{
In Vitro Multiparametric Cellular Analysis by Micro Organic Charge-modulated Field-effect Transistor Arrays
}

\author{
Andrea Spanu ${ }^{1}$, Annalisa Bonfiglio ${ }^{1}$ \\ ${ }^{1}$ Department of Electrical and Electronic Engineering, University of Cagliari
}

\section{Corresponding Author}

Andrea Spanu

andrea.spanu@unica.it

\section{Citation}

Spanu, A., Bonfiglio, A. In Vitro Multiparametric Cellular Analysis by Micro Organic Charge-modulated Fieldeffect Transistor Arrays. J. Vis. Exp. (175), e62907, doi:10.3791/62907 (2021).

\section{Date Published}

September 20, 2021

DOI

$10.3791 / 62907$

URL

jove.com/video/62907

\section{Abstract}

Modern electrophysiology has been constantly fueled by the parallel development of increasingly sophisticated tools and materials. In turn, discoveries in this field have driven technological progress in a back-and-forth process that ultimately determined the impressive achievements of the past 50 years. However, the most employed devices used for cellular interfacing (namely, the microelectrode arrays and microelectronic devices based on transistors) still present several limitations such as high cost, the rigidity of the materials, and the presence of an external reference electrode. To partially overcome these issues, there have been developments in a new scientific field called organic bioelectronics, resulting in advantages such as lower cost, more convenient materials, and innovative fabrication techniques.

Several interesting new organic devices have been proposed during the past decade to conveniently interface with cell cultures. This paper presents the protocol for the fabrication of devices for cellular interfacing based on the organic chargemodulated field-effect transistor (OCMFET). These devices, called micro OCMFET arrays (MOAs), combine the advantages of organic electronics and the peculiar features of the OCMFET to prepare transparent, flexible, and reference-less tools with which it is possible to monitor both the electrical and the metabolic activities of cardiomyocytes and neurons in vitro, thus allowing a multiparametric evaluation of electrogenic cell models.

\section{Introduction}

In vivo monitoring of electroactive cells, such as neurons and cardiomyocytes, represents a valid and powerful approach in fundamental research applications for the human brain, functional connectivity studies, pharmacology, and toxicology. The tools usually employed for such studies are mainly based on microelectrode arrays (MEAs) $)^{1,2,3,4,5}$ and increasingly more efficient and powerful field-effect devices $(\text { FEDs })^{6,7,8,9,10,11,12}$. These two families of devices allow the real-time monitoring and stimulation of the electrical activity of neurons and cardiomyocytes and are usually 
characterized by robustness, ease of use, and reliability. These features make MEAs and FEDs the gold standard for electrophysiological applications, being currently employed to interface with standard cellular cultures, organotypic brain slices, and tridimensional organoids ${ }^{13,14,15,16}$. Despite their widespread use and their impressive features, MEAs and FEDs present some limitations such as high cost, the rigidity of the materials, and the presence of a usually bulky reference electrode, which has to be placed in the measurement liquid environment and is necessary for proper operation of the devices.

To explore alternative solutions for cellular interfacing, much effort has been invested in the past decade into the study of electronic devices based on organic materials and innovative fabrication techniques ${ }^{17}$. Among the several organic devices studied to address the aforementioned limitations, a peculiar organic transistor called OCMFET has been recently proposed as a valid alternative to MEAs and FEDs $^{18}$. In addition to the standard features offered by the organic electronics technology, such as low-cost materials and fabrication techniques, optimal mechanical and chemical properties, optical transparency, and biocompatibility, the OCMFET also offers an ultra-high charge sensitivity (due to its double-gated structure) without the need of an external reference electrode. Moreover, this organic sensor has the remarkable capability of sensing different analyte/physical parameters, depending on the specific functionalization of its sensing area, which is separated from the transistor area $^{19,20}$. All these features can be conveniently exploited for the acquisition of different parameters within a cellular culture. In particular, in addition to being able to detect the neuronal/cardiac electrical activity, it is also possible to exploit the ultra-high $\mathrm{pH}$ sensitivity offered by the peculiar double-gated structure of the OCMFET by using a simple physical functionalization ${ }^{21}$ to reliably monitor the slight local $\mathrm{pH}$ variations caused by cellular metabolic activity.

In in vitro cell biosensing, the monitoring of cellular metabolic activity is a powerful indicator of the state of the culture and can be used to assess the cellular response to various stimuli, such as drug administration and electrical stimulation $^{22,23}$. Moreover, in the specific case of neural applications, monitoring both the electrical and the metabolic activities is of great interest, particularly in pharmacology and toxicology 24 . With the intent of conveniently addressing the requirements of modern in vitro electrophysiology while at the same time offering all the advantages of the OCMFET, a device called Micro OCMFET Array (MOA) has been recently introduced. The MOA is an OCMFET-based array with specialized sensing areas specifically designed for in vitro cellular interfacing, enabling the multiparametric analysis of electrogenic cells cultures. In particular, two MOA channels have bigger sensing areas to maximize their sensitivity and can be selectively functionalized to monitor specific parameters of interest, such as the $\mathrm{pH}$ variations of the culture medium. The other OCMFETs in the structure act as extracellular electrical activity sensors. Figure 1 shows the structure of a 16 channel MOA. This capability, combined with the absence of an external reference electrode, makes the MOA a very interesting tool for in vitro applications. This work presents the step-by-step fabrication protocol of a multisensing MOA for the in vitro detection of the electrical and metabolic activities of neurons and cardiomyocytes. Figure 2 shows the main fabrication steps, the materials used, and the device structure.

\section{Protocol}

All applicable international, national, and/or institutional guidelines for the care and use of animals were followed. All 
efforts were made to reduce the number of animals for the project and to minimize their suffering.

\section{Preparation of the developing solution, the etching solutions, the organic semiconductor solution, and the photolithographic masks}

1. Prepare the developing solution by diluting $\mathrm{NaOH}$ pellets in deionized water at a concentration of $175 \mathrm{mM}$.

NOTE: This is an exothermic reaction. If a plastic container is used, keep agitating the container until all pellets are completely dissolved.

2. Prepare the titanium etching solution by diluting hydrofluoric acid (HF) in deionized water (1 part of concentrated $48 \% \mathrm{HF}, 49$ parts deionized water).

CAUTION: Hydrofluoric acid can easily penetrate the skin, causing severe damage to deep tissue layers. Rapid neutralization of HF is necessary to prevent tissue destruction, which may continue for days and result in severe injury or even death. The risks associated with HF are dependent on the concentration and the duration of contact with the acid. Use only under a fume hood using a face shield. Double gloving is also strongly recommended.

3. Prepare the gold etching solution by mixing iodine, potassium iodide, and deionized water (for $250 \mathrm{~g}$ of solution, use $200 \mathrm{~mL}$ of deionized water, $20 \mathrm{~g}$ of $\mathrm{KI}, 5 \mathrm{~g}$ of 12 ). Stir the solution at room temperature for $1 \mathrm{~h}$ and leave it resting overnight before use.

4. Prepare the semiconductor solution by dissolving $6,13-$ bis(triisopropylsilylethynyl) pentacene (TIPS Pentacene) in anisole ( $1 \%$ in weight) and gently stirring for $2 \mathrm{~h}$ on a hot plate at $80{ }^{\circ} \mathrm{C}$.

NOTE: Keep agitating this solution. Use amber glass vials and/or store them in low light conditions.
5. Prepare the desired photolithographic mask set with a vector graphics software. Prepare 5 masks for the entire process: the mask for the patterning of the floating gates (FGs); the mask for the opening of the vias and the sensing areas for the electrophysiological recordings; the mask for the self-alignment process; the mask for the patterning of the source, drain and control gate top contact; and the mask for the plasma activation of the $\mathrm{pH}$ channels.

NOTE: Depending on the necessary resolution and the specific photolithographic setup, different kinds of masks could be used. In the case of the proposed devices (which have a maximum lateral resolution of $40 \mu \mathrm{m}$ ), simple plastic flexible masks have been bought at a local photocopy shop.

\section{Substrate selection and preparation}

1. Cut a $6 \times 6 \mathrm{~cm}^{2}$ square of $250 \mu \mathrm{m}$ polyethylene terephthalate (PET) from a pristine PET sheet.

NOTE: Start with a slightly bigger substrate than the final device to have wide enough margins to allow for manipulation with standard lab tweezers without damaging it.

2. Inspect the substrate with an optical microscope to exclude the presence of deep grooves and scratches. Carefully select the less scratched substrates as bigger imperfections may lead to the failure of the final device.

3. Rinse the PET substrates with acetone, isopropyl alcohol, and deionized water (in this order) and dry them using streams of nitrogen. Store the substrates in clean plastic Petri dishes/containers. 


\section{FG: titanium deposition}

1. Pre-clean the substrates with plasma oxygen ( $30 \mathrm{~s}$ at 100 W) and place them on the substrate holder inside the vacuum chamber of the thermal evaporator.

2. Place $60 \mathrm{mg}$ of titanium in the crucible, close the shutter, and pump down the evaporation chamber until it reaches a vacuum level lower than $10^{-6}$ Torr. Increase the power of the evaporator until the crucible glows red and wait for $30 \mathrm{~s}$. Open the shutter, increase the power to $60 \%$ (or until the crucible glows bright white), and wait for $60 \mathrm{~s}$. Close the shutter and turn down the power.

3. Remove the substrates from the evaporator; clean them using acetone, isopropyl alcohol, and deionized water; and dry them using streams of nitrogen. Perform a second oxygen plasma treatment $(60 \mathrm{~s}$ at $200 \mathrm{~W})$ to slightly oxidize the titanium surface.

\section{FG patterning}

1. Place one substrate at a time on the spin coater placed inside a fume hood. Deposit $4 \mathrm{~mL}$ of photoresist on the substrate using a disposable plastic pipette. Use the following spin coating parameters to obtain a $2 \mu \mathrm{m}$-thick photoresist layer: spin speed: 3000 rpm; spin time: $45 \mathrm{~s}$; acceleration time: $0.5 \mathrm{~s}$; deceleration time: $0.5 \mathrm{~s}$.

2. Soft bake the photoresist by placing the substrate on a hot plate $\left(70^{\circ} \mathrm{C}\right.$ for $\left.5 \mathrm{~min}\right)$. Store the substrate inside an aluminum foil-wrapped Petri dish/plastic container to avoid direct light exposure.

NOTE: Avoid the suggested bake temperature $\left(100{ }^{\circ} \mathrm{C}\right.$ for $50 \mathrm{~s}$ ) to prevent substrate deformation. However, baking at a lower temperature for a longer time ensures good results.
3. Place the device in a bromograph and position the plastic photolithographic mask with the desired FG layout onto the substrate. Expose to ultraviolet (UV) light from the top for $1 \mathrm{~min}$, and carefully remove the mask, taking care to minimize the lateral movements of the mask over the substrate to avoid scratching it.

4. Plunge the substrate for $5 \mathrm{~s}$ in a glass container filled with the developing solution (step 1.1). Quickly rinse it in deionized water and dry it under nitrogen. Use an optical microscope to look for underdeveloped/ overdeveloped spots in the substrate. Repeat the immersion of the substrate in developing solution in case of underdevelopment.

5. Etch the exposed titanium by submerging it in the titanium etching solution (step 1.2) for $15 \mathrm{~s}$, rinse it with deionized water, and dry it using nitrogen. Optically inspect the substrate and remove the photoresist using acetone. Rinse the substrate with isopropyl alcohol and deionized water, and dry it with nitrogen.

\section{Gate dielectric deposition}

1. Prepare the deposition chamber of the Parylene coater by distributing $2 \mathrm{~mL}$ of the adhesion promoter (silane 3-(trimethoxysilyl)propyl methacrylate) on the deposition chamber walls using a laboratory wipe. Place $300 \mathrm{mg}$ of Parylene $\mathrm{C}$ dimer (corresponding to a final thickness of $150 \mathrm{~nm}$ ) onto the Parylene coater. Set the lower pressure value to $7 \mathrm{mbar}$ and the higher pressure value to $10 \mathrm{mbar}$. After the deposition, clean the substrates with acetone, isopropyl alcohol, and deionized water, and dry them with nitrogen. 


\section{Opening of the sensing areas of the OCMFET for electrical activity recording and formation of the vias to access the back of the FGs}

1. Deposit the photoresist on the substrates using the same parameters of steps 4.1 and 4.2 .

2. Place the device in a bromograph and position the plastic photolithographic mask onto the substrate for the vias (circular openings with a diameter of $50 \mu \mathrm{m}$ over the sensing areas and $100 \times 100 \mu \mathrm{m}^{2}$ openings over the FGs away from the sensing areas (referred to as back contact of the FGs in Figure 1 and Figure 2)) under a stereoscopic microscope to improve the alignment precision. Expose to UV light from the top for $1 \mathrm{~min}$, and carefully remove the mask, taking care to minimize the lateral movements of the mask over the substrate to avoid scratching it.

NOTE: The vias on the side of the FGs away from the sensing area (shown as back contact of FGs in Figure 1 and Figure 2) are necessary for contact during the characterization of the transistor. Moreover, having electrical access to the FGs may be very useful for different types of functionalization (e.g., electrodeposition).

3. Develop the photoresist as previously described in step 4.4. Expose the substrate with the patterned photoresist (which acts as a mask here) to oxygen plasma (180 s at $200 \mathrm{~W}$ ) to remove the Parylene $\mathrm{C}$ from the sensing areas.

NOTE: The etching rate of Parylene $\mathrm{C}$ in an isotropic plasma cleaner at $200 \mathrm{~W}$ is approximately $90 \mathrm{~nm} / \mathrm{min}$. A slight over-etch is performed to further clean the sensing areas. The photoresist is also etched during the process. However, its thickness $(2 \mu \mathrm{m})$ is much higher than that of the Parylene C.
4. Place the substrates into a glass container filled with acetone inside the ultrasonic bath for $10 \mathrm{~s}$ to remove the photoresist completely. Rinse the substrates with acetone, isopropyl alcohol, and water and dry them with nitrogen.

NOTE: Using sonication instead of simply rinsing the substrates with acetone is crucial to prevent undesired folding and re-deposition of Parylene $\mathrm{C}$ fragments onto the surface of the sensing areas.

\section{Self-alignment of source and drain with the FG}

1. Deposit the photoresist on the substrates using the same parameters of steps 4.1 and 4.2. Place the device in a bromograph and position onto the substrate a plastic photolithographic mask with simple black rectangles that completely cover the transistor's areas. Expose to UV light for $1 \mathrm{~min}$ from both the top and the bottom, and carefully remove the mask, taking care to minimize the lateral movements of the mask over the substrate to avoid scratching it.

NOTE: With the double-sided exposure, the FGs act as photolithographic masks with respect to the bottom exposure, while the presence of the top mask ensures that only the photoresist present on the transistors' channel remains unexposed.

2. Develop the photoresist as previously described in step 4.4 .

\section{Gold deposition, channel formation, and patterning of the sources, drains, and control gates}

1. Clean the substrates with a gentle plasma treatment (30 $\mathrm{s}$ at $30 \mathrm{~W}$ ) to promote the adhesion of the metal on the 
Parylene $\mathrm{C}$ and place them on the substrate holder inside the vacuum chamber of the thermal evaporator.

2. Place $30 \mathrm{mg}$ of gold in the crucible, close the shutter, and pump down the evaporation chamber until it reaches $10^{-5}$ Torr. Increase the power of the evaporator until the crucible glows red and wait for $30 \mathrm{~s}$. Open the shutter, increase the power to $40 \%$ (or until the crucible glows bright white), wait for $60 \mathrm{~s}$, close the shutter, and turn down the power.

3. Place the substrates into an acetone container inside the ultrasonic bath for $10 \mathrm{~s}$ to lift off the photoresist, thus removing the gold from the transistors' channel. Rinse the substrates with acetone, isopropyl alcohol, and water and dry them with nitrogen. Deposit the photoresist on the substrates using the same parameters of steps 4.1 and 4.2 .

4. Place the device in a bromograph and position onto the substrate a plastic photolithographic mask with the desired sources, drains, and control gate layout. Expose to UV light for $1 \mathrm{~min}$ from the top, and carefully remove the mask, taking care to minimize the lateral movements of the mask over the substrate to avoid scratching it.

5. Develop the photoresist as described in step 4.4. Etch the exposed gold by submerging it in the gold etching solution (step 1.3) for $10 \mathrm{~s}$, rinse it with deionized water, and dry it out using nitrogen. Optically inspect the substrate and remove the photoresist using acetone. Rinse with isopropyl alcohol and deionized water and dry it with nitrogen.

\section{Deposition and activation of the Parylene $\mathrm{C}$ for pH sensing}

1. Deposit the photoresist on the substrates using the same parameters of steps 4.1 and 4.2 .

2. Place the device in a bromograph and position onto the substrate a plastic photolithographic mask with openings corresponding to the $\mathrm{pH}$-sensing areas of the OCMFETs. Expose to UV light for $1 \mathrm{~min}$ from the top, and carefully remove the mask, taking care to minimize the lateral movements of the mask over the substrate to avoid scratching it.

3. Develop the photoresist as described in step 4.4. Protect the whole device, except for the $\mathrm{pH}$-sensing areas, with polyimide insulation tape (see the Table of Materials). Deposit a layer of $500 \mathrm{~nm}$ of Parylene C (corresponding to $1 \mathrm{~g}$ of Parylene $\mathrm{C}$ dimer) on the substrate using the same parameters described in step 5.1.

NOTE: The total Parylene $\mathrm{C}$ thickness on the $\mathrm{pH}$-sensing areas is $650 \mathrm{~nm}$. No silane is required for this deposition.

4. Carefully remove the polyimide insulation tape. Expose the substrate to oxygen plasma ( $5 \mathrm{~min}$ and $30 \mathrm{~s}$ at 200 W) to activate the Parylene $\mathrm{C}$ on the $\mathrm{pH}$-sensing areas of the OCMFETs.

NOTE: The polyimide insulation tape is necessary here to limit the Parylene $\mathrm{C}$ deposition. In fact, a simple lift-off using the photoresist does not give positive results due to the almost pinhole-free nature of the conformal coating obtained with Parylene C.

5. Place the substrates into an acetone container inside the ultrasonic bath for $10 \mathrm{~s}$ to completely remove the photoresist. Rinse the substrates with acetone and isopropyl alcohol (no water) and dry them with nitrogen. 


\section{Semiconductor deposition, culturing chamber placing, and final cut-out of the device from the PET}

1. Place the substrates onto a hot plate at $50^{\circ} \mathrm{C}$. Cast a droplet $(1 \mu \mathrm{L})$ of semiconductor solution (step 1.4) onto each channel area, cover the whole substrate with a lid, and let it dry out under a chemical hood for $30 \mathrm{~min}$.

2. Prepare the culturing chamber by printing an acrylonitrile butadiene styrene ring with an internal radius of $15 \mathrm{~mm}$, a thickness of $1 \mathrm{~mm}$, and a height of $7 \mathrm{~mm}$ with a 3D printer. Glue the culturing chamber onto the central part of the substrate using polydimethylsiloxane (ratio of the curing agent: $15 \%$ by weight). Cut out the device from the PET either manually or using a laser cutter.

\section{Electrical characterization of transistors}

1. Characterize each transistor using a sourcemeter $^{18,19,20,21}$ (see the Table of Materials). NOTE: Both the output and the input characteristics should be measured to extrapolate the transistors' parameters (mainly the carriers' mobility, threshold voltage, ION/lOFF ratio, and subthreshold slope).

\section{Representative Results}

The potential of the MOA has been validated here for both electrical activity recordings and metabolic activity monitoring. The precise estimation of the capabilities of the device to detect extracellular action potentials was based on a thorough characterization with rat cardiomyocytes cultures (particularly in primary rat cardiomyocytes measured at 8 days in vitro $[\mathrm{DIV}])^{18}$. Figure $3 \mathrm{~A}$ shows a complete MOA with 16 OCMFETs. The top inset shows an example of a confluent rat cardiomyocyte culture adhering to the surface of the MOA.
To highlight their health, the cells have been immunostained for the sarcomeric protein, tropomyosin, after the recording session. The bottom inset shows a single cardiomyocyte signal measured with an OCMFET.

Interestingly, the device could detect spontaneous electrical activity and the activity induced upon the administration of different chemicals, as shown in Figure 3B. This validation was crucial to demonstrate the feasibility of using this approach for electrogenic cell interfacing. Because of the array configuration, the MOA also allowed for the reconstruction of the propagation velocity of the cardiac signal, thus demonstrating the suitability of the system for the study of cellular networks (Figure 3C). For further validation to determine the actual detection limit of the device, the MOA was also tested with striatal neurons $(21 \mathrm{DIV})^{18}$, with interesting results in terms of signal amplitude and the reliability of the recordings. As seen in Figure 3D, the OCMFET could amplify neuronal field potentials with remarkable stability, showing signal-to-noise ratios (SNRS) of up to 3.2 (in the same range as that of SNRs obtained with standard $M E A s^{25}$ ). The recording setup consisted of custom multichannel electronics for the transistor biasing and the signal readout and conditioning. Each channel for the electrical recording has a first stage consisting of an I/ $\mathrm{V}$ converter with a $1 \mathrm{M} \Omega$ feedback resistor and a 150 $\mathrm{Hz}-1.3 \mathrm{kHz}$ bandpass filter with a voltage gain of 110 . For all the presented measurements, the transistors were biased with $V_{D S}=V_{G S}=-1 \mathrm{~V}$. The $A / D$ conversion and the data visualization and storing were performed using a data acquisition board (see the Table of Materials). All the measurement sessions were conducted inside a Faraday cage to minimize the electrical, environmental noise on the system. 
As previously mentioned, by exploiting the simple physical functionalization presented in the protocol, it was possible to prepare highly sensitive $\mathrm{pH}$ sensors with a supernernstian response. Because of the presented fabrication approach, these $\mathrm{pH}$ devices could be integrated into an MOA and used to monitor the slight $\mathrm{pH}$ variations induced by the metabolic activity of primary hippocampal rat neurons ${ }^{26}$. In particular, as shown in Figure 4, only one of the two OCMFETs dedicated to low-frequency sensing was selectively functionalized to demonstrate the feasibility of the approach. This selective functionalization allowed the evaluation of the response of the two OCMFETs to chemically induced metabolic variations: in particular, a high metabolic state can be obtained using bicuculline (BIC), an inhibitor of GABA A receptors ${ }^{27}$, while a low metabolic state can be induced by the addition of tetrodotoxin (TTX), which eventually causes cellular death ${ }^{28}$. The recording setup consisted of the same custom multichannel electronics used for the electronic activity measurements.

Unlike the previous case, two dedicated channels were used to record the slow variations induced by the cellular metabolic activity. Each channel consisted of a simple circuit composed of two main blocks: an I/V converter with a $1 \mathrm{M} \Omega$ feedback resistor and a low-pass filter with a cut-off frequency of $10 \mathrm{~Hz}$. The transistors were biased with $V_{D S}=V_{G S}=-1 \mathrm{~V}$, and all the measurements were carried out inside a Faraday cage to minimize the impact of external noise on the recordings (this is a particularly important aspect considering the low current fluctuations induced by the cellular metabolic activity). During the experiments, the cultures were maintained in a low-buffered culture medium, and the whole system was placed in a controlled environment $\left(37^{\circ} \mathrm{C}\right.$ and a continuous $\mathrm{CO}_{2}$ /air flux). As expected, only the current of the $\mathrm{pH}$ sensitive OCMFET could be modulated by the addition of 25 $\mu \mathrm{M}$ BIC. This was further confirmed by the induction of the current variation by the corresponding variation of the cellular metabolic activity.

The same experiment was repeated after the addition of $10 \mu \mathrm{M}$ TTX, which resulted in a gradual slowing down of the cellular metabolism. Following the addition of the TTX, neither the $\mathrm{pH}$-sensitive OCMFET nor the $\mathrm{pH}$-insensitive one showed any response, thus demonstrating the efficacy of the approach. These results demonstrate the effectiveness of the proposed functionalization and its relative stability for up to 2 weeks. An important conclusion that can be drawn from the proposed experiments (both the electrical activity and the metabolic activity) is that it is possible to prepare different kinds of sensors by selectively functionalizing different OCMFETs within the same culturing area. This aspect represents a non-trivial achievement in biosensing for cellular applications because being able to monitor different parameters within the same cell culture is crucial for better characterization of the complexity of those biological systems. 


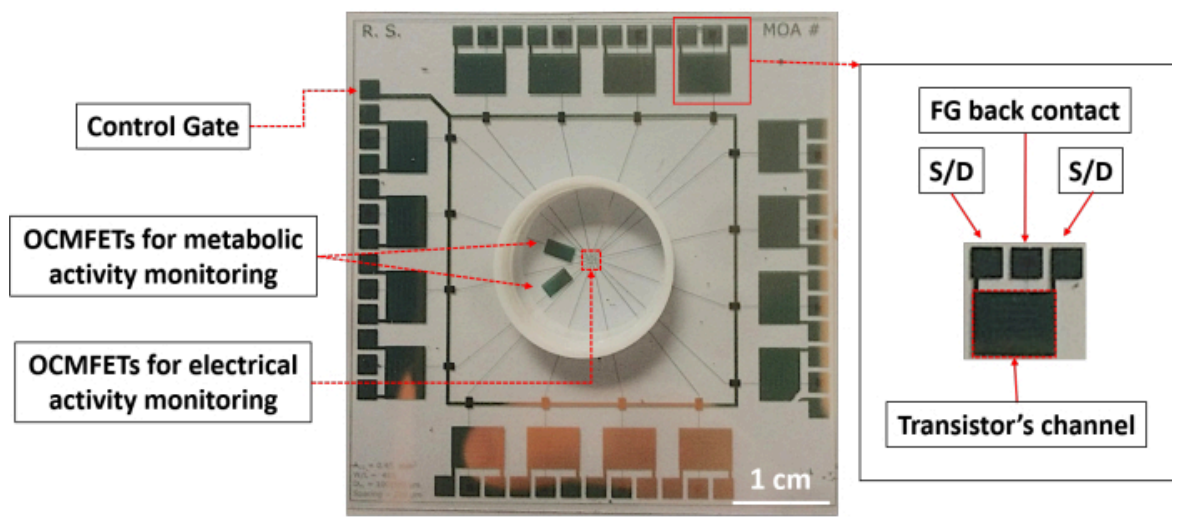

Figure 1: Top view of a 16 channel MOA for metabolic and electrical monitoring of electroactive cells. Scale bar $=1$ $\mathrm{cm}$. Abbreviations: OCMFETs = organic charge-modulated field-effect transistors; FG = floating gate; S/D = source/drain; MOA = micro OCMFET array. Please click here to view a larger version of this figure. 
A

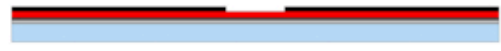

B

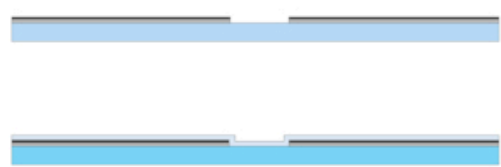

D

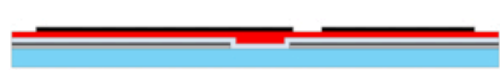

E

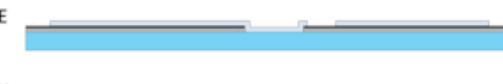

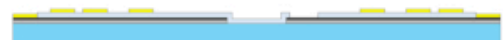

G
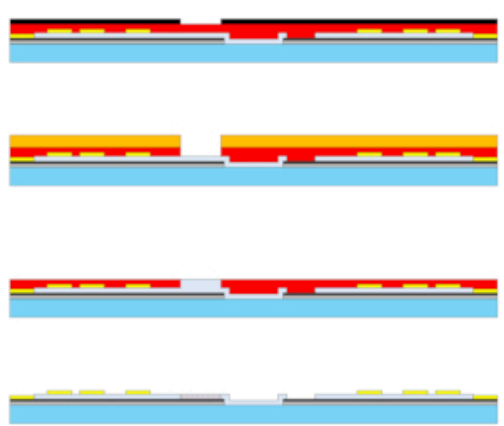

TIPS

Native TiO

Activated Par C 매매

ABS ring

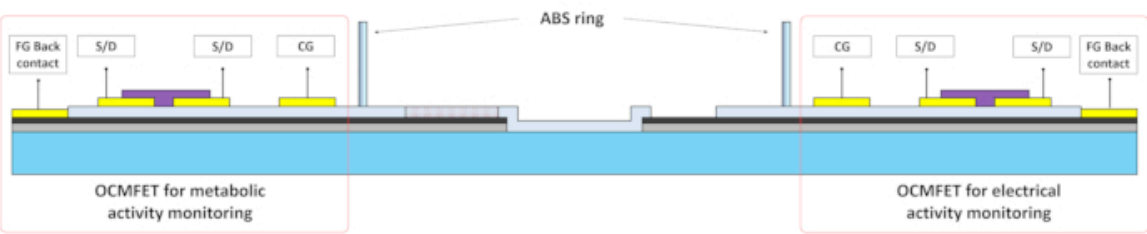

Figure 2: Main fabrication steps of an MOA for metabolic and electrical monitoring of electroactive cells. (A and

B) The evaporated Ti film is patterned using a standard photolithographic process to prepare the floating gate of the OCMFETs. (C) Deposition of $15 \mathrm{~nm}$ of Parylene C. This layer, together with the native Ti oxide, acts as the gate dielectric of the transistors. (D and E) The Parylene $C$ layer is patterned using plasma oxygen treatment. A patterned photoresist layer is used to selectively expose the sensing areas for the electrical recordings and the floating gate back contacts. (F) Patterning of the Au top contacts, namely the source, drain, control gate, and floating gate back contact. A self-alignment technique is used to improve the electrical performance of the device. (G-I) Deposition of the second layer of Parylene $\mathrm{C}$ on the sensing area of the OCMFETs for metabolic activity monitoring. After the oxygen plasma exposure, this layer will act as the $\mathrm{pH}$-sensitive membrane $(\mathbf{J})$. (K) Cross-section of a complete MOA (with materials) after the deposition of the organic semiconductor (TIPS Pentacene) and the culture chamber positioning. Abbreviations: OCMFETs $=$ organic charge-modulated field-effect transistors; FG = floating gate; $S / D=$ source/drain; $M O A=$ micro OCMFET array; $C G=$ control gate; $\mathrm{PET}=$ polyethylene terephthalate; Par $\mathrm{C}=$ Parylene $\mathrm{C} ; \mathrm{TIPS}=6,13$-bis(triisopropylsilylethynyl) pentacene; $\mathrm{ABS}=$ acrylonitrile butadiene styrene. Please click here to view a larger version of this figure. 


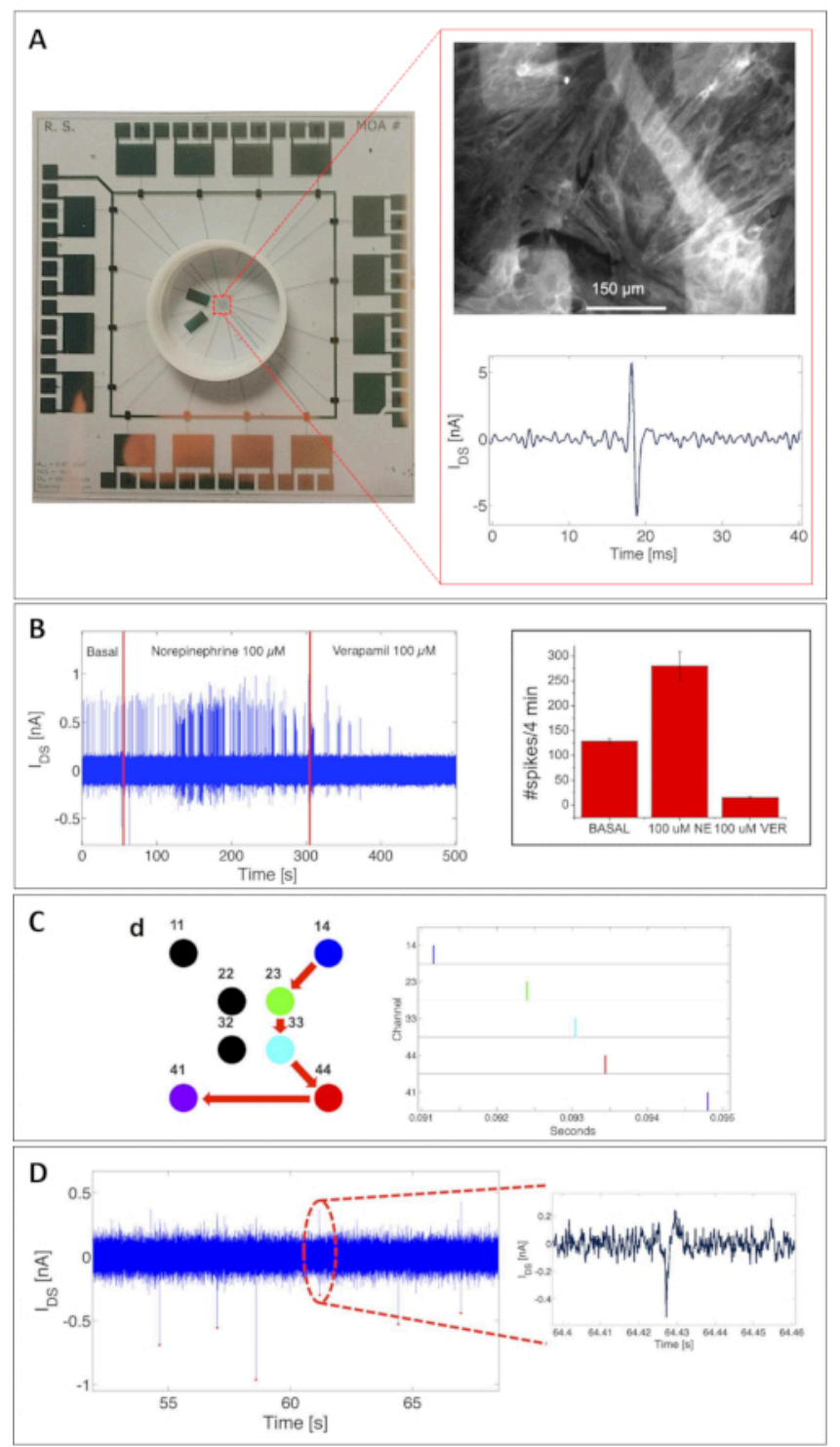

Figure 3: Cellular electrical activity recordings with an MOA. (A) A confluent culture of rat cardiomyocytes (8 DIV) adhering to the surface of an MOA, fixed after a recording session and immunostained for the sarcomeric protein, tropomyosin (upper inset). Bottom inset: example of a single cardiomyocyte signal measured with an OCMFET. Scale bar $=150 \mu \mathrm{m}$. (B) Chemical tuning of the electrical activity of a cardiomyocyte culture. The activity acceleration resulted from the addition of $100 \mathrm{mM}$ norepinephrine, while suppression resulted from the addition of $100 \mathrm{mM}$ verapamil. Left: beating frequency modulation; right: statistics on 5 OCMFETs-average and standard deviation: spike-count on 4 min of basal (129 \pm 4.6), norepinephrine-mediated ( $280 \pm 28.6)$ and verapamil-mediated activity $(15 \pm 1.9)$. (C) Reconstruction of the propagation of a cardiac signal. Right: raster plot of the spontaneous activity of the culture indicating the propagation of the signal from site 14 to site 41 (right). (D) Action potentials of striatal cells from rat embryo (21 DIV). This figure has been modified 
from ${ }^{18}$. Abbreviations: OCMFET = organic charge-modulated field-effect transistor; MOA = micro OCMFET array; NE = norepinephrine; VER = verapamil; DIV = days in vitro. Please click here to view a larger version of this figure.
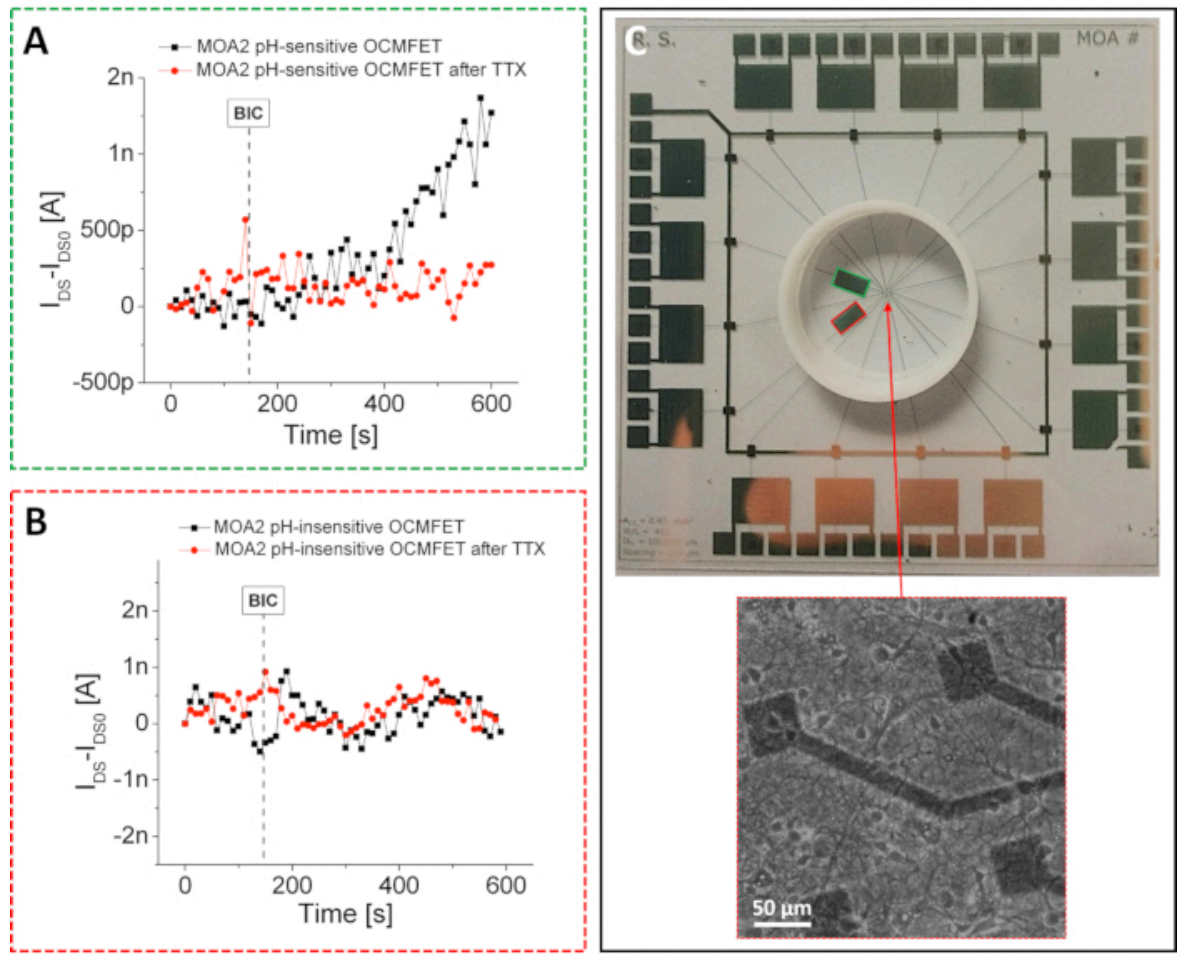

Figure 4: Metabolic activity recordings with an MOA. Response of the (A) $\mathrm{pH}$-sensitive and (B) $\mathrm{pH}$-insensitive channels of an MOA to the addition of $25 \mu \mathrm{M} \mathrm{BIC}$ before and after the addition of $10 \mu \mathrm{M}$ of TTX. After the TTX addition, the behavior of the $\mathrm{pH}$-sensitive channel becomes similar to that of the $\mathrm{pH}$-insensitive one. In particular, no current variation can be observed after the BIC addition due to the TTX-induced cellular death. (C) MOA for metabolic activity recordings. The pHsensitive and the $\mathrm{pH}$-insensitive OCMFETs are outlined in green and red, respectively. Inset: healthy hippocampal neurons cultured onto the device after 15 DIV. Scale bar $=50 \mu \mathrm{m}$. This figure has been modified from ${ }^{26}$. Abbreviations: OCMFET = organic charge-modulated field-effect transistor; MOA = micro OCMFET array; $\mathrm{BIC}=$ bicuculline; TTX = tetrodotoxin; DIV = days in vitro. Please click here to view a larger version of this figure.

\section{Discussion}

Unlike previous methods for the fabrication of OCMFETs for cellular applications ${ }^{18,29}$, the proposed method is specifically designed to prepare MOAs that can simultaneously detect electrical and metabolic cellular activity. Moreover, this approach to achieve $\mathrm{pH}$ sensitivity has the advantage of being compatible with standard fabrication protocols and does not involve any chemical modification of the sensing area (this aspect ensures the biocompatibility of the whole device). The $\mathrm{pH}$ sensitivity is achieved using the same material used as a gate dielectric (i.e., the biocompatible Parylene $\mathrm{C}$ ), making this approach fast and reproducible. 
The final result of this approach is a flexible, transparent, low-cost, and multisensing organic tool for in vitro cellular applications. The fact that this can be obtained using a single transistor structure and a simple physical modification of the sensing area adds to the advantages offered by the use of organic electronic materials and methods. Moreover, because the transduction principle of the OCMFET does not strictly depend on the specific semiconductor or FG material, the whole process can be modified and upscaled depending on the specific application.

A critical aspect of the proposed technique is related to the reproducibility of the plasma activation technique. To obtain consistent results, both the Parylene $C$ thickness and its etching rate must be controlled. Frequent calibration of the Parylene $\mathrm{C}$ deposition process and the plasma cleaner are absolutely necessary. Other critical aspects, which also contribute to the reproducibility of the process, are the careful handling of the device and the deposition of the organic semiconductor. A simple drop-casting technique was used here, which intrinsically poses reproducibility limitations. To minimize those issues, as described in protocol step 10.1, the same amount of semiconductor solution should be used every time, and the solvent evaporation should be standardized as much as possible. Keeping a constant temperature using a hot plate and covering the substrate after each droplet deposition will help in slowing down the evaporation process. To further minimize this problem, the deposition technique (e.g., using an inkjet printing method) could be switched ${ }^{30}$.

A limitation of the proposed protocol stems from the nature of the functionalization of the OCMFET for $\mathrm{pH}$ sensing. The stability of the $\mathrm{pH}$ sensors is limited to a few weeks ${ }^{26}$. However, the stability window of the proposed approach is sufficiently large to cover the standard incubation times needed for neuronal culture growth (2-3 weeks). Other types of sensing area functionalization should be considered for longer experiments. The fabrication protocol uses a dedicated back contact, allowing electrical access to the FGs. This contact, which is left floating during the normal operation of the device, can be exploited for the electrical characterization of the device and the functionalization of the sensing areas using different techniques (e.g., electrodeposition).

This procedure represents a convenient way to prepare a multi-sensing device for cellular applications without the need for expansive materials or cleanroom facilities. Despite the performance and stability limitations due to the employment of an organic semiconductor and physical (not chemical) functionalization of the sensing area, similar approaches could be used to prepare low-cost (and potentially disposable), mechanically flexible, and optically transparent sensors and biosensors, which can provide researchers in cellular biology, tissue engineering, and neuroscience with novel specialized tools for studying cellular systems in vitro.

\section{Disclosures}

The authors have no conflicts of interest to declare.

\section{Acknowledgments}

The authors acknowledge funding from the European Union's Horizon 2020 research and innovation programme under grant agreement No. 882897-Search\&Rescue project and the PON project "TEX-STYLE" ARS01_00996, PNR 2015-2020.

\section{References}

1. Hubel, D. H. Tungsten microelectrode for recording from single units. Science. 125 (3247), 549-550 (1957). 
2. Verzeano, M., Negishi, K., Angeles L. Neuronal activity in cortical and thalamic networks. A study with multiple microelectrodes. Journal of General Physiology,. 43 (6), 177-195 (1960).

3. Thomas, C. A. Jr, Springer, P. A., Loeb, G. E., BerwaldNetter, Y., Okun, L. M. A miniature microelectrode array to monitor the bioelectric activity of cultured cells. Experimental Cell Research. 74 (1), 61-66 (1972).

4. Grattarola, M., Martinoia, S. Modeling the neuronmicrotransducer junction: from extracellular to patch recording. IEEE Transactions on Biomedical Engineering, . 40 (1), 35-41 (1993).

5. Wallace K., Strickland J. D., Valdivia P., Mundy W. R., Shafer T. J. A multiplexed assay for determination of neurotoxicant effects on spontaneous network activity and viability from microelectrode arrays. NeuroToxicology. 49, 79-85 (2015).

6. Bergveld, P. Development, operation, and application of the tool for electrophysiology. IEEE Transactions on Biomedical Engineering. 19 (5), 342-351 (1972).

7. Bergveld, P., Wiersma, J., Meertens, H. Extracellular potential recordings by means of a field effect transistor without gate metal, called OSFET. IEEE Transactions on Biomedical Engineering. 23 (2), 136-144 (1976).

8. Fromherz, P., Offenhausser, A., Vetter, T., Weis, J. A neuron-silicon junction: a Retzius cell of the leech on an insulated-gate field-effect transistor. Science. 252 (5010), 1290-1293 (1991).

9. Martinoia, S. et al. Development of ISFET array-based microsystems for bioelectrochemical measurements of cell populations. Biosensors and Bioelectronics. 16 (9-12), 1043-1050 (2001).
10. Heer, F. et al. CMOS microelectrode array for the monitoring of electrogenic cells, Biosensors and Bioelectronics,. 20 (2), 358-366 (2004).

11. Berdondini, L. et al. Active pixel sensor array for high spatio-temporal resolution electrophysiological recordings from single cell to large scale neuronal networks. Lab on a Chip. 9 (18), 2644-2651 (2009).

12. Maccione, A. et al. Multiscale functional connectivity estimation on low-density neuronal cultures recorded by high-density CMOS micro electrode arrays. Journal of Neuroscience Methods. 207 (2), 161-171 (2012).

13. Kibler A. B., Jamieson B. G., Durand D. M. A high aspect ratio microelectrode array for mapping neural activity in vitro. Journal of Neuroscience Methods. 204 (2), 296-305 (2012).

14. Frega, M., Tedesco, M., Massobrio, P., Pesce, M., Martinoia, S. Network dynamics of 3D engineered neuronal cultures: a new experimental model for in-vitro electrophysiology. Scientific Reports,. 4, 5489 (2014).

15. Zuo, L., Yu, S., Briggs, C. A., Kantor, S., Pan, J. Y. Design and fabrication of a three-dimensional multielectrode array for neuron electrophysiology. Journal of Biomechanical Engineering. 139 (12) (2017).

16. Spanu, A. et al. A three-dimensional micro-electrode array for in-vitro neuronal interfacing. Journal of Neural Engineering. 17 (3), 036033 (2020).

17. Spanu, A., Martines, L., Bonfiglio, A. Interfacing cells with organic transistors: a review of in vitro and in vivo applications, Lab on a Chip., 21 (5), 795-820 (2021).

18. Spanu, A. et al. An organic transistor-based system for reference-less electrophysiological monitoring of excitable cells. Scientific Reports. 5, 8807 (2015). 
19. Viola, F. A., Spanu, A., Ricci, P. C., Bonfiglio, A., Cosseddu, P. Ultrathin, flexible and multimodal tactile sensors based on organic field-effect transistors. Scientific Reports. 8, 8073 (2018).

20. Napoli, C. et al. Electronic detection of DNA hybridization by coupling organic field-effect transistor-based sensors and hairpin-shaped probes. Sensors. 18 (4), 990 (2018).

21. Spanu, A. et al. A reference-less $\mathrm{pH}$ sensor based on an organic field effect transistor with tunable sensitivity. Organic Electronics. 48, 188-193 (2017).

22. Lundgaard, I. et al. Direct neuronal glucose uptake heralds activity-dependent increases in cerebral metabolism. Nature Communications. 6, 6807 (2015).

23. Zhang, Y. S. et al. Multisensor-integrated organs-onchips platform for automated and continual in situ monitoring of organoid behaviors. Proceedings of the National Academy of Sciences of the United States of America. 114 (12), E2293-E2302 (2017).

24. $\mathrm{Yu}, \mathrm{H}$. et al. A novel design of multifunctional integrated cell-based biosensors for simultaneously detecting cell acidification and extracellular potential. Biosensors and Bioelectronics. 24 (5), 1462-1468 (2009).

25. Maccione, A. et al. A novel algorithm for precise identification of spikes in extracellularly recorded neuronal signals. Journal of Neuroscience Methods. 177 (1), 241-249 (2009).

26. Spanu, A., Tedesco, M. T., Martines, L., Martinoia, S., Bonfiglio, A. An organic neurophysiological tool for neuronal metabolic activity monitoring. APL Bioengineering. 2 (4), 046105 (2018).
27. Díaz-García, C. M. et al. Neuronal stimulation triggers neuronal glycolysis and not lactate uptake. Cell Metabolism. 26 (2), 361-374.e4 (2017).

28. Xie, Y., Dengler, K., Zacharias, E., Wilffert, B., Tegtmeier, F. Effects of the sodium channel blocker tetrodotoxin (TTX) on cellular ion homeostasis in rat brain subjected to complete ischemia. Brain Research. 652 (2), 216-224 (1994).

29. Caboni, A., Orgiu, E., Barbaro, M., Bonfiglio, A. Flexible organic thin-film transistors for $\mathrm{pH}$ monitoring. IEEE Sensors Journal. 9 (12), 1963-1970 (2009).

30. Fraboni, B., Bonfiglio, A., Basiricò, L. Inkjet printing of transparent, flexible, organic transistors. Thin Solid Films. 520, 1291-1294 (2011). 